\title{
Review Article \\ Fire-Needle Moxibustion for the Treatment of Knee Osteoarthritis: A Meta-Analysis
}

\author{
Yidan Wang, ${ }^{1}$ Xiaohua Xie, ${ }^{2}$ Xiaoyue Zhu, ${ }^{1}$ Minjie Chu, ${ }^{1}$ Yihua Lu, ${ }^{1}$ Tian Tian, \\ Xun Zhuang, ${ }^{1}$ and Liying Jiang ${ }^{1}$ \\ ${ }^{1}$ Department of Epidemiology, School of Public Health, Nantong University, Nantong, Jiangsu 226009, China \\ ${ }^{2}$ Department of Endodontics, Institute of Hard Tissue Development and Regeneration, \\ The Second Affiliated Hospital of Harbin Medical University, Harbin, Heilongjiang 150001, China \\ Correspondence should be addressed to Liying Jiang; j_meili@126.com
}

Received 13 January 2016; Revised 11 April 2016; Accepted 12 May 2016

Academic Editor: Antonella Fioravanti

Copyright (c) 2016 Yidan Wang et al. This is an open access article distributed under the Creative Commons Attribution License, which permits unrestricted use, distribution, and reproduction in any medium, provided the original work is properly cited.

\begin{abstract}
Objectives. The aim of this study was to evaluate the effectiveness of fire-needle moxibustion as an intervention in the treatment of knee osteoarthritis (KOA). Methods. An updated meta-analysis of randomized controlled trials (RCTs) on fire-needle moxibustion in treating KOA was conducted by searching PubMed, Embase, the Cochrane Library, Web of Science, Wanfang database, and the Chinese Medical Database (CNKI) since their inception through March 2016. The meta-analysis was performed using RevMan 5.3. Results. Thirteen RCTs were identified in the systematic study which consisted of 1179 participants. Fire-needle moxibustion treatment group had a statistical significance on recovery rate as well as recovery and marked-improvement rate compared with control group. Subgroup analysis indicated that there was significant difference between fire-needle moxibustion group and control group. However, GRADE analysis indicated that the quality of evidence for all outcomes was relatively low. Only two of 13 studies reported adverse reactions (difficulty in movement and intolerance of cold). Conclusion. This meta-analysis suggests that fire-needle moxibustion is more effective than control group in symptom management of KOA. Further high quality trials should be conducted to evaluate the effectiveness of fire-needle moxibustion on KOA.
\end{abstract}

\section{Introduction}

Osteoarthritis (OA), the most common joint bone disease, is characterized by the chronic degenerative changes of joint structure including cartilage surfaces and subchondral bone [1]. As the major part of weight-bearing peripheral joint, the knee is the most frequently affected site [2]. The structural changes result in pain, stiffness, swelling, and tenderness and then reduce physical function and affect the quality of life of patients [3]. About $10-13 \%$ of the causes of disability for people aged 60 years and above are attributable to knee osteoarthritis (KOA) [4]. It has become a major public health problem as KOA is associated with advancing age and universal obesity $[5,6]$.

The occurrence and development of $\mathrm{OA}$ are related to multiple aetiological factors such as genetics, environmental factors, and biomechanical components. Presently, the symptomatic approach is clinically the premier choice $[7,8]$.
The conventional treatment of KOA includes medicine and surgical treatment [9]. Although these conventional treatments are usually recommended for the relief of pain, severe adverse events attached to these therapies were reported [10, 11]. Acupuncture and moxibustion, as mainstream complementary and alternative treatments in nonpharmacological therapy, have been widely accepted by patients across the world to relieve pain, improve function, and restrain the progression of KOA [12].

Filiform-needle acupuncture, warm-needle moxibustion, and fire-needle moxibustion are three main types of acupuncture and moxibustion. Topical acupoints such as Heding, Xiyan, Xuehai, Liangqiu, and Ashi were usually selected. Filiform-needle acupuncture is a modality of traditional acupuncture applied directly to the skin surface with sterile stainless steel [13]. Warm-needle moxibustion involves stimulating acupuncture points with burning moxa 


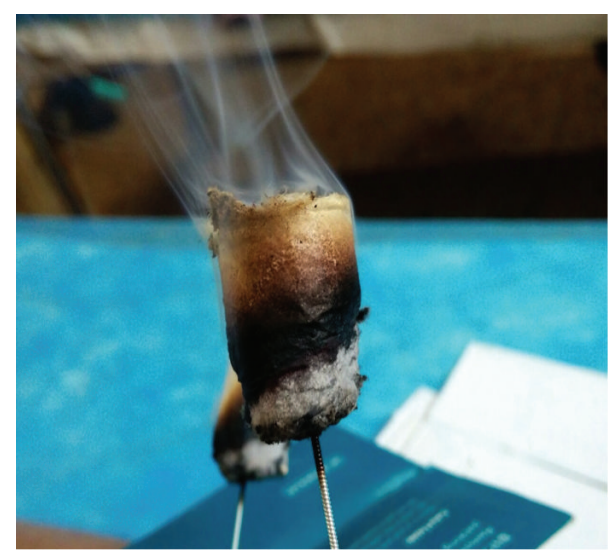

FIGURE 1: The photo of warm-needle moxibustion.

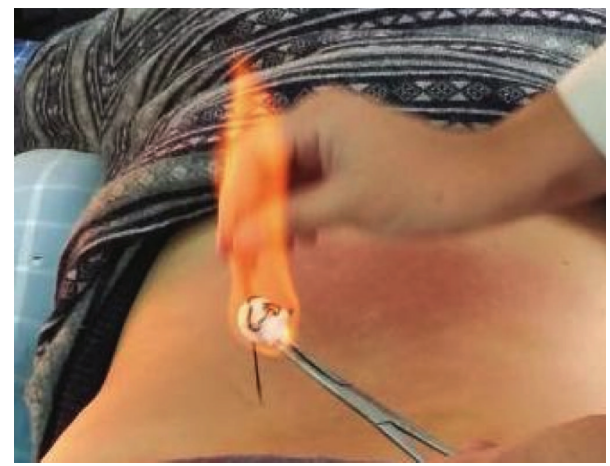

FIGURE 2: The photo of fire-needle moxibustion.

containing the herb Artemisia vulgaris [14] (Figure 1). Fireneedle moxibustion is an ancient method of external therapy that combines acupuncture with moxibustion. The acupoints of fire-needle therapy will be the same as those in the filiform needle and warm needle except for needling depth, acupuncture manipulation, and needle retention time. The tip of the fire needle is round so as to increase the contact area with the lesions. What is more, the fire needle has the property of high temperature resistance (Figure 2). In the operation, needle retention time of the fire needle is less than that of filiform needle and warm needle. Skilled Traditional Chinese Medicine (TCM) practitioners use the needle to prick the selected acupoints by depth of $0.3-0.5$ cun and then remove the needle swiftly. Unless the needle holes are bleeding, practitioners are not supposed to press [15].

At present, filiform-needle acupuncture and warmneedle moxibustion have been confirmed as effective treatments of KOA $[16,17]$. Several randomized controlled trials (RCTs) have proved that fire-needle moxibustion could relieve pain and improve the prognosis of KOA. However, the efficacy of fire-needle moxibustion has not been systematically assessed [18]. Therefore, we undertook a systematic review and meta-analysis to assess the current evidence for the effect and safety of fire-needle moxibustion in treating OA.

\section{Methods}

2.1. Search Strategy. An Internet-based search was performed through PubMed, Embase, the Cochrane Library, Web of Science, Wanfang database, and the Chinese Medical Database (CNKI) since their inception through March 2016. The publication language was limited to English and Chinese. Search terms in Chinese included "Huo Zhen", "gu guan jie yan", and "xi gu guan jie yan". The following search terms in English were used: "fire needling", "fire needle", "osteoarthritis", "OA", "arthritis", “joint disease", and the corresponding free terms. The search was restricted to studies of human participants.

2.2. Inclusion and Exclusion Criteria. Inclusion criteria were as follows. (1) Types of study: studies were eligible if they were RCTs. (2) Types of participants: the research samples included participants in accordance with the KOA diagnosis criteria from the American College of Rheumatology (ACR) and the Guiding Principles of Clinical Research on New Drugs for TCM [19, 20]. (3) Types of interventions: studies that compared fire-needle moxibustion with routine acupuncture or warm-needle moxibustion were eligible. No restriction was made regarding selection of acupoints. (4) Outcome measure: clinical recovery rate and recovery and marked-improvement rate were the major outcome of assessment. Their pain intensity was assessed according to the Visual Analog Scale (VAS), Index of Severity for Osteoarthritis (ISOA), and/or the scale of the Western Ontario and McMaster Universities Arthritis Index (WOMAC). If available, safety served as the secondary outcome.

The following exclusion criteria were applied: (1) the study was of nonrandomized or uncontrolled trials; (2) the study did not describe intervention methods clearly; (3) the study was lacking sample size and data of related index; (4) the study or data of the research was reported repeatedly.

2.3. Data Extraction. Two reviewers (Yidan Wang and Xiaoyue Zhu) evaluated the obtained studies independently according to a preconfigured form. Characteristics of the included studies such as date of publication, author, participants' quantity, interventions, outcome measures, and results were presented in Table 1. Disagreements were resolved by discussion with a third reviewer (Liying Jiang).

2.4. Risk of Bias and Quality Assessment. The Cochrane Risk of Bias Tool was used to evaluate the methodological quality of the included studies (version 5.1.0) [21]. The tool included seven domains: random sequence generation, allocation concealment, blinding of participants and personnel, blinding of outcome assessment, incomplete outcome data, selective report, and other sources of bias. Each item was judged by the following criteria: low (low risk of bias), unclear (uncertain risk of bias), and high (high risk of bias). Two reviewers (Yidan Wang and Xiaoyue Zhu) checked the aspects of each included study independently. GRADEpro 3.6.1 software was applied to assess the quality of evidence for all outcomes, and the results were summarized in Table 2. 


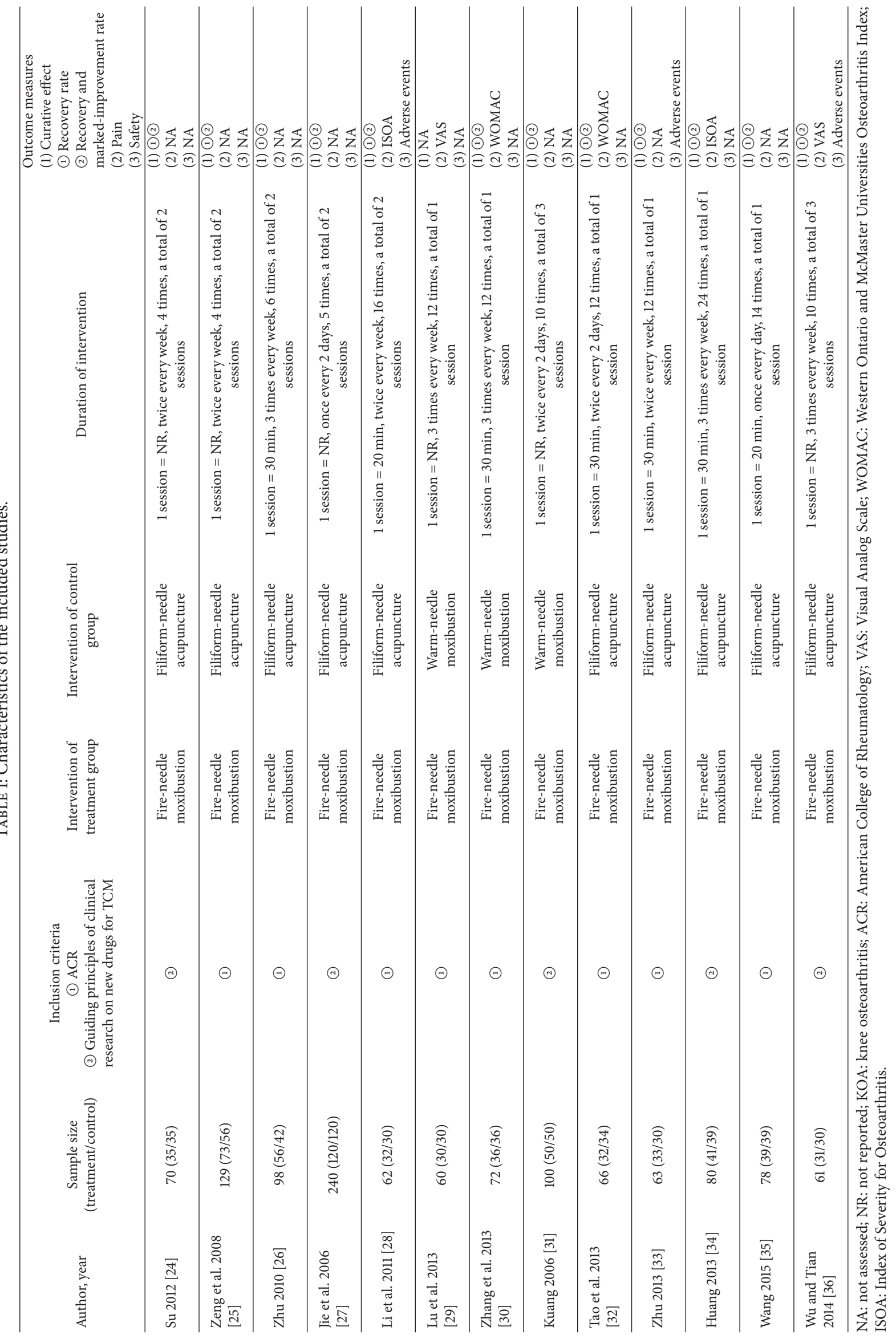


TABLE 2: Grade quality of evidence of fire-needle moxibustion treatment for KOA.

\begin{tabular}{|c|c|c|c|c|}
\hline \multirow{2}{*}{ Outcome } & \multicolumn{2}{|c|}{ Effect } & \multirow{2}{*}{$\begin{array}{l}\text { Number of participants } \\
\text { (studies) }\end{array}$} & \multirow{2}{*}{$\begin{array}{l}\text { Quality of the evidence } \\
\text { (GRADE) }\end{array}$} \\
\hline & Relative effect (95\% CI) & Absolute effect (95\% CI) & & \\
\hline Recovery rate & $\begin{array}{c}\mathrm{RR} 1.56 \\
\text { (1.34 to } 1.81) \\
\end{array}$ & $\begin{array}{l}162 \text { more per } 1000 \\
\text { (from } 98 \text { more to } 234 \text { more) }\end{array}$ & $\begin{array}{c}1114 \\
\text { (12 studies) } \\
\end{array}$ & $\begin{array}{l}\oplus \oplus \ominus \ominus \\
\text { Low }^{1,2}\end{array}$ \\
\hline $\begin{array}{l}\text { Recovery and } \\
\text { marked-improvement rate }\end{array}$ & $\begin{array}{c}\mathrm{RR} 1.50 \\
\text { (1.36 to } 1.64) \\
\end{array}$ & $\begin{array}{l}250 \text { more per } 1000 \\
\text { (from } 180 \text { more to } 319 \text { more) }\end{array}$ & $\begin{array}{c}1051 \\
\text { (11 studies) }\end{array}$ & $\begin{array}{l}\oplus \oplus \ominus \ominus \\
\text { Low }^{1,2}\end{array}$ \\
\hline Pain & & $\begin{array}{c}\text { SMD } 0.72 \text { SD lower } \\
\text { (1.23 lower to } 0.22 \text { lower) }\end{array}$ & $\begin{array}{c}390 \\
\text { (6 studies) }\end{array}$ & $\begin{array}{c}\oplus \ominus \ominus \ominus \\
\text { Very low }{ }^{1,2,3}\end{array}$ \\
\hline
\end{tabular}

${ }^{1}$ None of the trials were blinded; most of them did not mention randomization process and allocation concealment.

${ }^{2}$ Published evidence is limited due to a small number of trials, all of which are showing benefits.

${ }^{3}$ Confidence intervals with minimal overlap; the heterogeneity is significant.

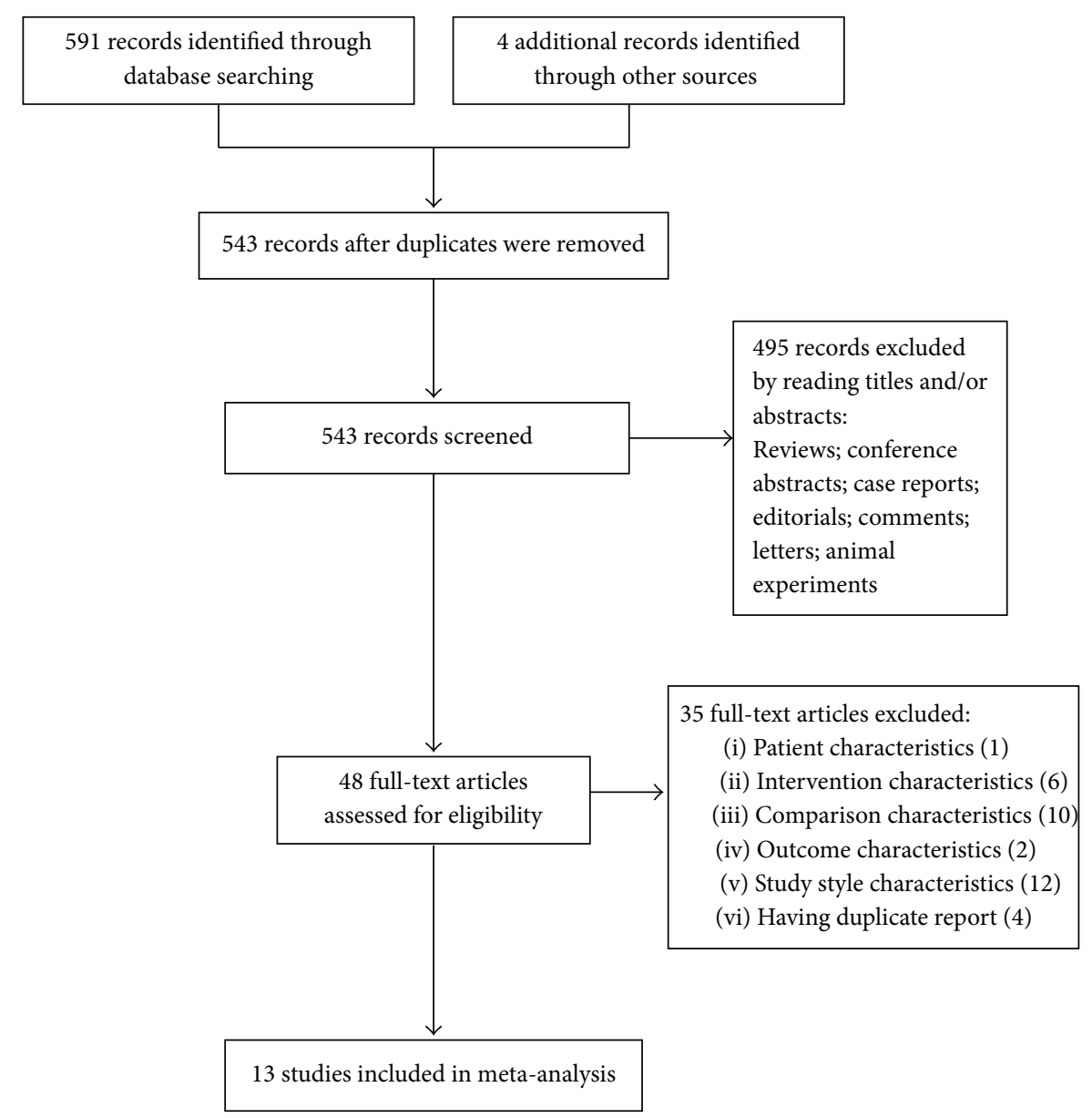

FIGURE 3: Flowchart of study search.

2.5. Statistical Analysis. All statistical analyses were performed by using Review Manager version 5.3 (the Nordic Cochrane Centre, the Cochrane Collaboration, Copenhagen, Denmark). For dichotomous outcomes, we calculated relative risk (RR) with 95\% confidence interval (CI). For continuous outcomes, standardized mean difference (SMD) with 95\% confidence interval (CI) was used to present the effect size. Heterogeneity was assessed by applying a chi-squared test. $I^{2}$ was considered to indicate a substantial level of heterogeneity. A random-effects model was used if $I^{2}>50 \%$. We constructed the funnel plot by Stata 12.0 to assess the potential publication bias when 10 or more trials were included in the meta-analysis [22]. A subgroup analysis should be conducted because different types of pain scale would lead to statistical heterogeneity [23].

\section{Results}

3.1. Study Selection and Characteristics. A flowchart of search selection was shown in Figure 3. We identified 595 potentially 

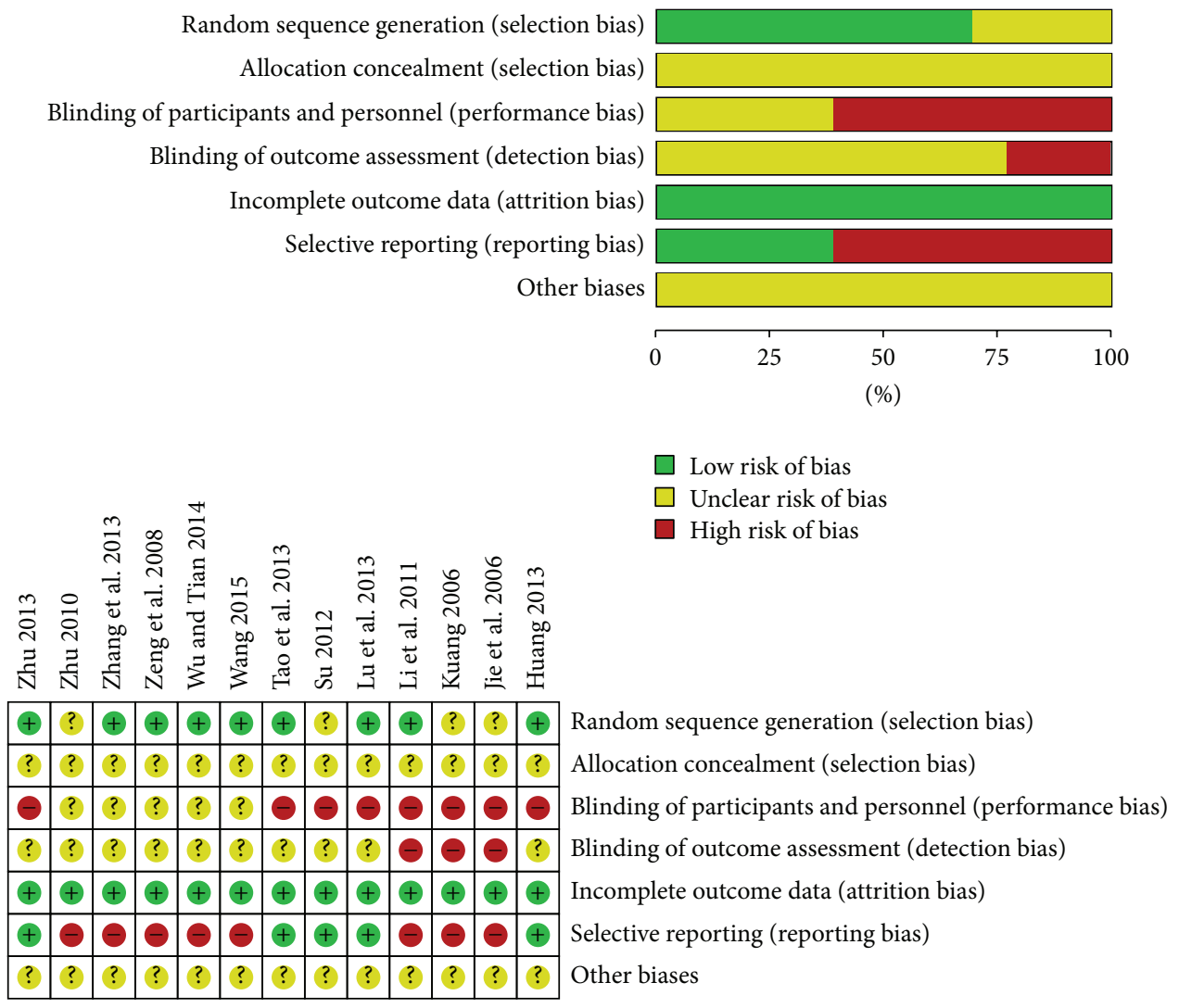

FIgURE 4: Plots of bias risk.

relevant articles. After 52 duplicate records were removed, we screened the remaining 543 records for eligibility and excluded 495 publications based on titles and/or abstracts, mainly because they were reviews, conference abstracts, case reports, editorials, comments, letters, and animal experiments. 48 full-text publications were obtained for further review. 35 articles were excluded which were irrelevant to the principle of PICOS (Patients-Intervention-ComparisonOutcome-Study Style): the characteristics of patients (1 study), intervention (6 studies), comparison (10 studies), outcome (2 studies), study style (12 studies), and duplicate reports (4 studies). Therefore, only 13 RCTs were included in our meta-analysis [24-36]. Characteristics of included studies were shown in Table 1.

All these included studies were carried out in the Chinese population and published in a Chinese journal. A total of 1179 cases were enrolled in this meta-analysis. Sample size varied from 61 to 240 . The participants were randomly assigned to fire-needle group and control group with their mean age ranging from 46 to 73 . The duration of the treatment ranged from 2 weeks to 2 months. All the studies used fire-needle moxibustion as the intervention of treatment group. The details of interventions of control group were summarized as follows: 10 RCTs used filiform-needle acupuncture [32-36] and 3 RCTs used warm-needle moxibustion as the control [29-31]. All the included studies reported both recovery rate and recovery and marked-improvement rate to evaluate curative effect. Six studies had reported pain intensity: two studies used ISOA [28, 34], two studies adopted VAS [29, 36], and two studies employed WOMAC [30, 32]. The adverse events were only reported in two studies $[28,33]$, while those 11 studies did not demonstrate this [24-27, 29-32, 34-36].

3.2. Risk of Bias. Risk of bias in those included studies was summarized in Figure 4. Randomization was mentioned in all trials. However, 4 studies did not report details of adequate sequence generation $[24,26,27,31]$. The method of allocation concealment was not described in any of these trials. Three trials were considered to have a low risk of bias for blinding of outcome assessment [27, 28, 31]. Since both fire-needle patients and providers clearly were aware of the treatment, blinding of providers or patients was an impossible criterion to set for fire-needle moxibustion therapy. There was no dropout in any of the included studies, and all studies reported complete outcome data. Eight studies had a high risk of reporting bias [25-28, 30, 31, 35, 36].

3.3. The Recovery Rate. 12 studies [24-28, 30-36] reported the recovery rate with a total sample of 1114 participants (575 in fire-needle moxibustion treatment group and 539 in the control group). A fixed-effects model was performed to analyze the data according to the heterogeneity test $\left(I^{2}=\right.$ $0 \%, P=0.98)$. The meta-analysis of these studies showed that there was a statistically significant difference between fire-needle group and control group in the recovery rate 


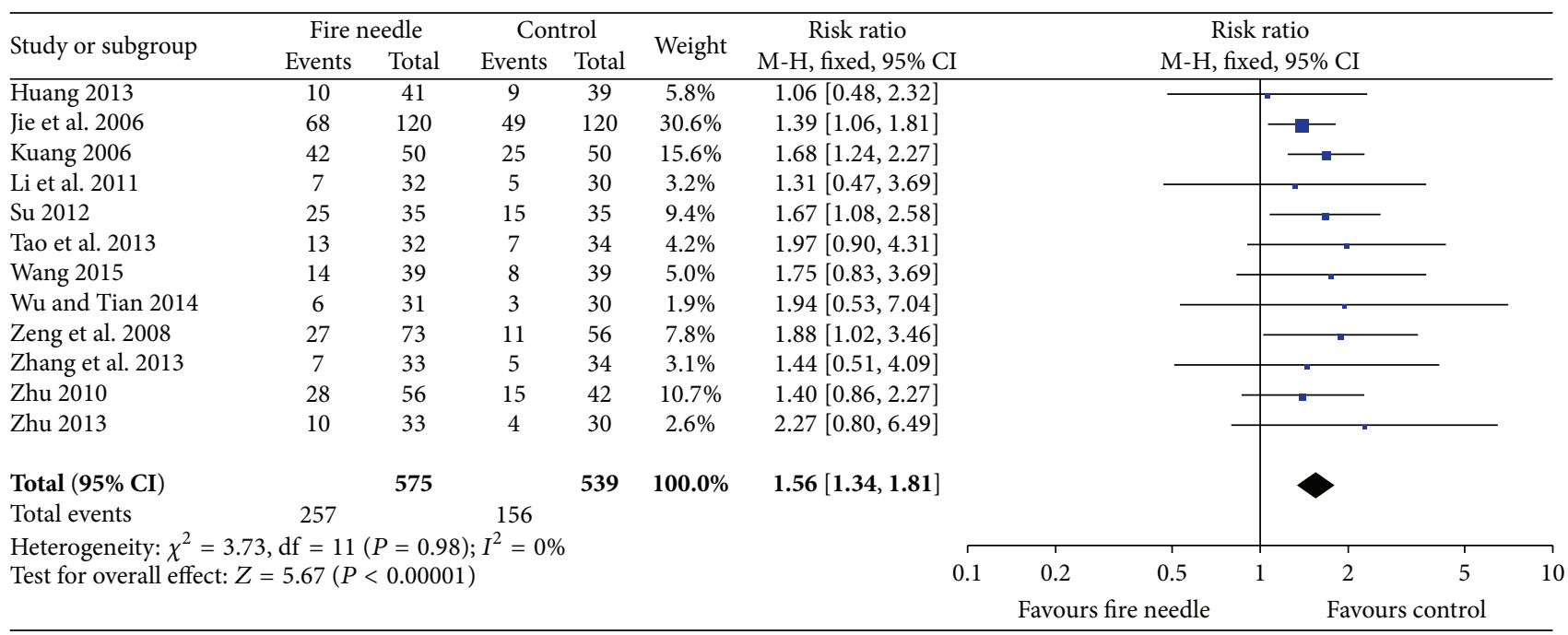

FIGURE 5: Forest plot of recovery rate.

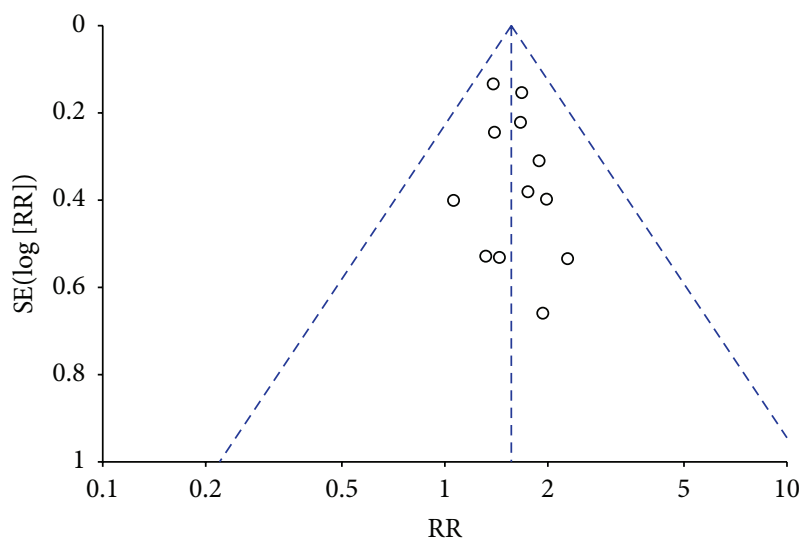

FIGURE 6: Funnel plot of recovery rate.

( RR $=1.56,95 \%$ CI: 1.34-1.81, $P<0.00001$; Figure 5). A funnel plot based on studies on recovery rate was generated to detect the potential publication bias, and it manifested a significant asymmetry in Figure 6 (Egger's test, $P=0.047$ ).

3.4. The Recovery and Marked-Improvement Rate. Eleven studies involving 1051 participants (542 in fire-needle moxibustion treatment group and 509 in the control group) reported the recovery and marked-improvement rate. The data were analyzed using a fixed-effects model in accordance with the acceptable heterogeneity $\left(I^{2}=20 \%, P=\right.$ $0.25)$. The meta-analysis showed that there was a significant high recovery and marked-improvement rate on fire-needle moxibustion treatment compared with the control group $(\mathrm{RR}=1.50,95 \% \mathrm{CI}: 1.36-1.64, P<0.00001$; Figure 7$)$. A funnel plot analysis of 11 studies comparing fire-needle moxibustion treatment with control group on the recovery and marked-improvement rate was performed to assess the publication bias. All points in Figure 8 were asymmetrical, which indicated that publication bias might have existed (Egger's test, $P=0.039$ ).

3.5. Pain Relief. Six studies measured pain intensity using VAS [29, 36], ISOA [28, 34], and WOMAC [30, 32]. A random-effects model was used because of significant heterogeneity $\left(I^{2}=83 \%, P<0.0001\right)$. After pooling, the results indicated that fire-needle moxibustion treatment might have a better effect on pain relief than conventional treatment (SMD $=-0.72,95 \% \mathrm{CI}:-1.23-0.22, P=0.005$; Figure 9). We failed to conduct a funnel plot to detect publication bias on pain relief because of the insufficient number of studies.

3.6. Adverse Effects. Three RCTs assessed adverse effects [28, $33,36]$, while those ten RCTs did not report this. Two of the three RCTs reported difficulty in movement and intolerance of cold $[28,33]$.

\section{Discussion}

Fire-needle moxibustion, as a kind of traditional medicine, has been widely used in China [37-39]. Although a growing number of studies reporting fire-needle moxibustion for treating KOA patients ranged from case report studies to cohort studies to randomized controlled trials, there was no systematic review specially referring to its effectiveness in the treatment of KOA. Therefore, we conducted this metaanalysis to evaluate the efficacy of fire-needle moxibustion for KOA.

Our systematic review of 13 RCTs included 1179 participants and presumably revealed that fire-needle moxibustion in the treatment of KOA might increase the recovery rate $(\mathrm{RR}=1.56,95 \% \mathrm{CI}: 1.34-1.81, P<0.00001)$ and recovery and marked-improvement rate $(\mathrm{RR}=1.50,95 \% \mathrm{CI}: 1.36-$ $1.64, P<0.00001)$ compared with control group. In addition, the pain intensity score in the fire-needle moxibustion group was significantly lower than that in the control group 


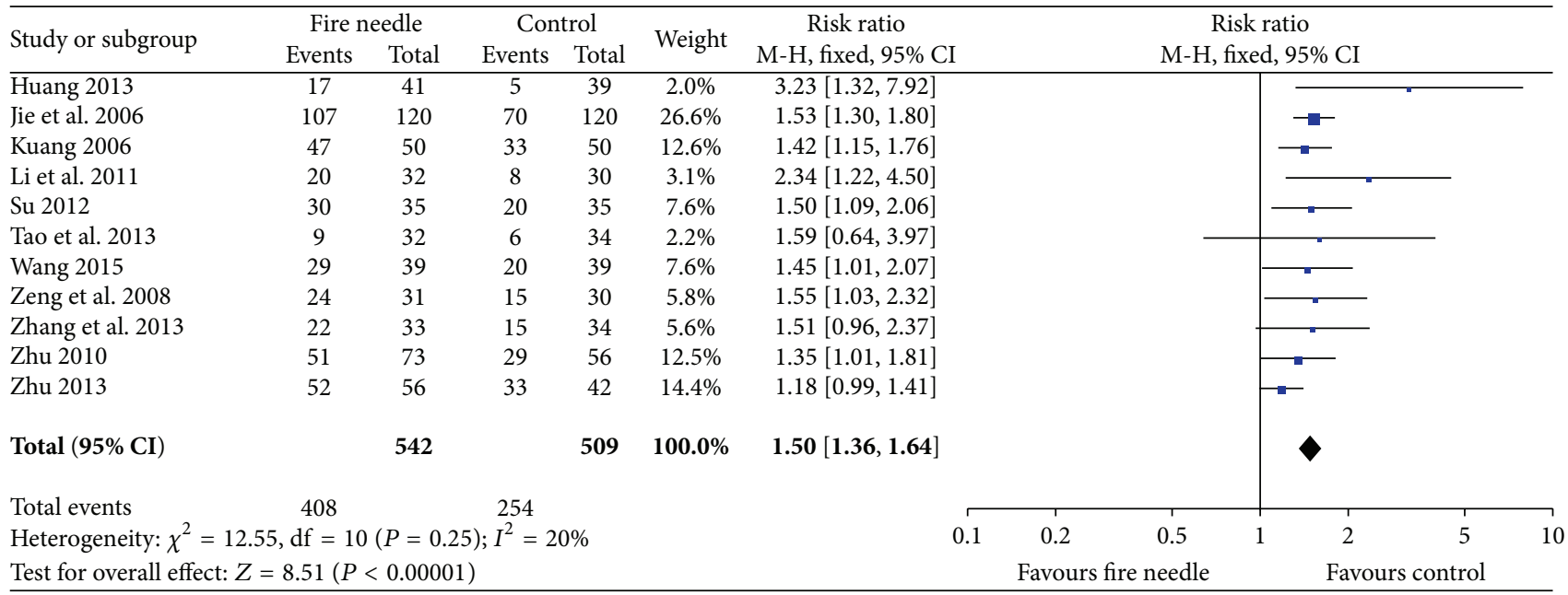

FIGURE 7: Forest plot of recovery and marked-improvement rate.

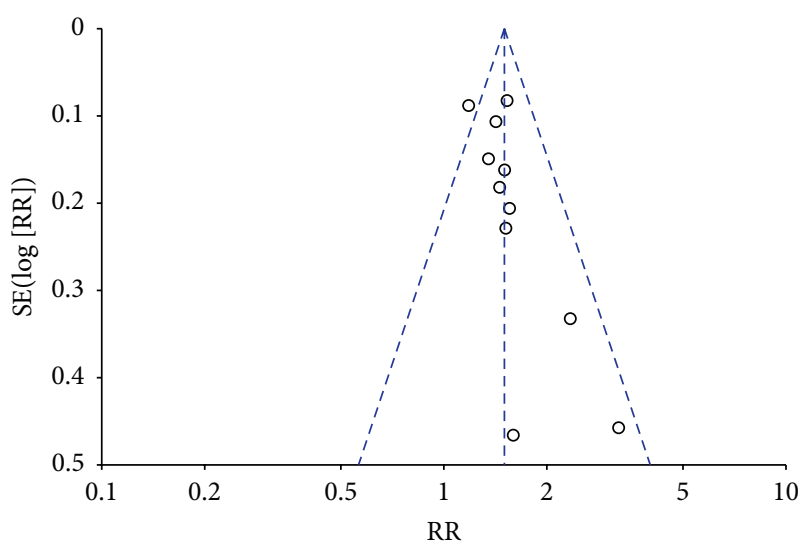

FIGURE 8: Funnel plot of recovery and marked-improvement rate.

$(\mathrm{SMD}=-0.72,95 \% \mathrm{CI}:-1.23-0.22, P=0.005)$. However, GRADE profile indicated that the quality of evidence for all outcomes was relatively low. Taking into account the limited sample size and poor methodological quality of the included trials, it was difficult to draw robust conclusions.

Fire-needle moxibustion has a synergistic effect of heat from moxibustion and stimulation on acupoints in promoting calcification, improving blood circulation, and eliminating blood stasis [40,41]. With the increasing use of fire-needle moxibustion, recent studies have reported the potential adverse events including allergies, burns, infection, difficulty in movement, and intolerance of cold [28, 33, 42]. Considering that most of the included studies have not demonstrated the adverse events and follow-up, we are unable to adequately assess the effectiveness and safety. More information is needed to better evaluate the adverse effects of fire-needle moxibustion.

The diversity in quality of the trials might lead to methodological heterogeneity, while the difference in PICO (patients, intervention, control, and outcomes) might lead to clinical heterogeneity. Subgroup analysis is an important approach to exploring the heterogeneity of treatment effects in RCTs. Given that different types of pain scale would lead to statistical heterogeneity, we conducted a subgroup analysis. However, it is difficult to assess this heterogeneity in terms of needling depth, acupuncture manipulation, and needle retention time because those detailed pieces of information are unavailable.

Our study provided the opportunity for foreign readers to recognize the advantages and exploited a new field for the research and application of fire-needle moxibustion therapy. What is more, we strictly searched the literature and thoroughly extracted and analyzed the data in order to ensure the credibility of our results.

There are some limitations that need to be considered. Firstly, the primary limitation is the poor methodological quality of the included studies. Randomization, allocation concealment, and blinding should be reported clearly, as these are the core standards of a well-designed RCT [43, 44]. The included RCTs in our study all mentioned randomization. Nevertheless, most of the trials lacked details regarding adequate sequence generation and allocation concealment. It is hard to blind the providers and patients owing to the nature of acupuncture and moxibustion. The low quality of the included studies suggested that the results should be interpreted with caution. Secondly, all trials included in the analysis were conducted in the Chinese population, which might limit the generalizability of the results. Thirdly, all trials claimed positive effects of fire-needle moxibustion in the treatment of KOA, implying that publication bias may have existed. As negative findings are less likely to be published, nonpositive studies have been inevitably missed [45]. Last but not least, we failed to generate a funnel plot for pain relief to detect potential publication bias due to the limited number of included trials.

In conclusion, the results of this meta-analysis indicate that fire-needle moxibustion may be more effective in symptom management of KOA when compared with control 


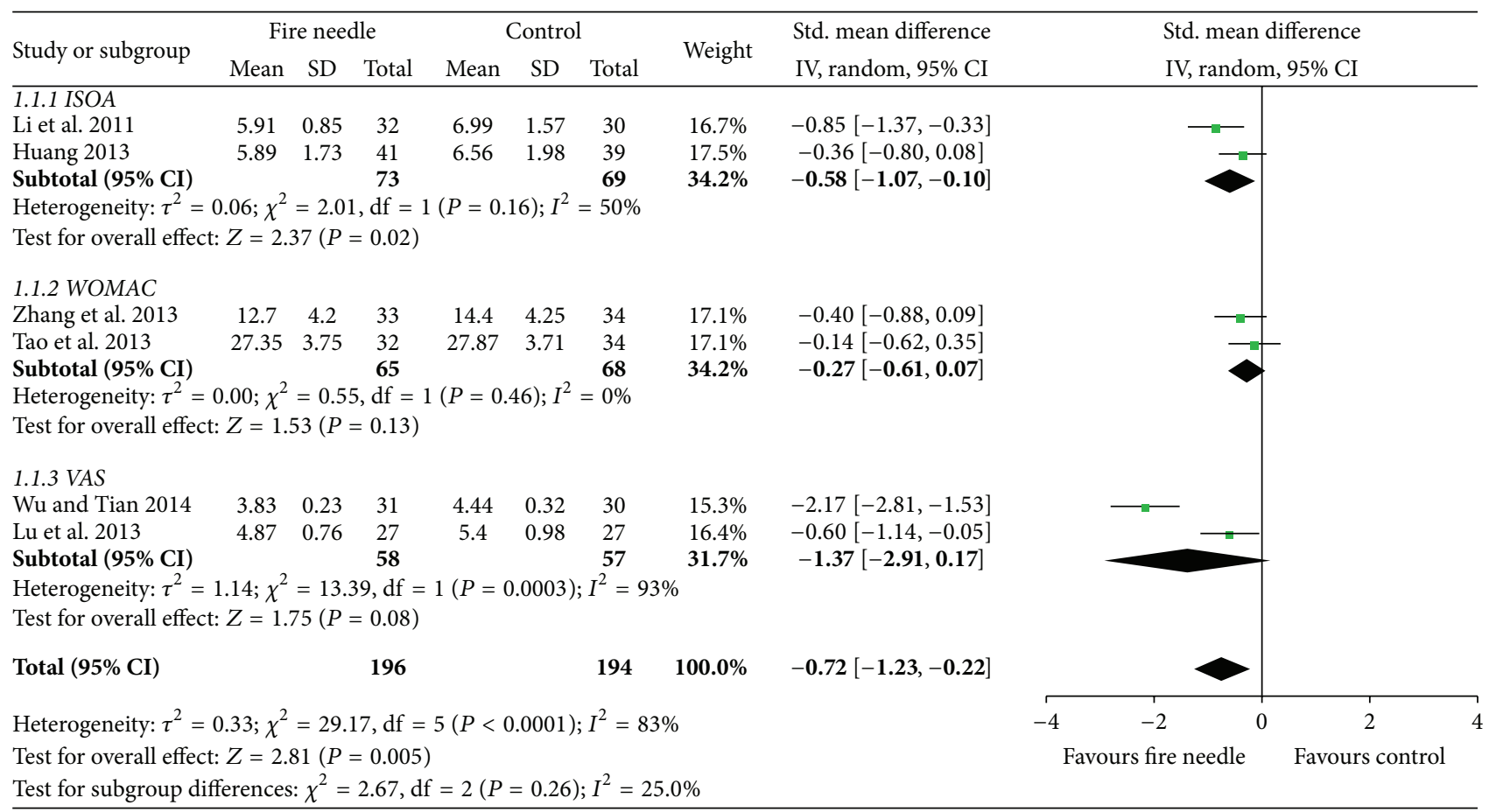

FIGURE 9: Fire-needle group and control group on pain.

group. However, the findings should be interpreted cautiously because of the insufficient number of rigorously designed studies. More rigorously designed and higher quality trials with larger sample size are necessary for better elucidating the effectiveness of fire-needle moxibustion on KOA.

\section{Disclosure}

This study did not warrant institutional review board approval as no human subjects were involved.

\section{Competing Interests}

The authors declared that there are no potential competing interests with respect to the research, authorship, and/or publication of this paper.

\section{Authors' Contributions}

Yidan Wang drafted the protocol and wrote the final paper. Liying Jiang contributed to the research design and made critical revisions. Xiaoyue Zhu was responsible for the statistical design of the trial and wrote portions of the statistical methods, data handling, and monitoring sections. All authors read and approved the final paper.

\section{Acknowledgments}

This study was supported by Nantong Municipal Science and Technology Bureau (MS12015114).

\section{References}

[1] D. T. Felson, R. C. Lawrence, P. A. Dieppe et al., "Osteoarthritis: new insights. Part 1: the disease and its risk factors," Annals of Internal Medicine, vol. 133, no. 8, pp. 635-646, 2000.

[2] S. Haaz and S. J. Bartlett, "Yoga for arthritis: a scoping review," Rheumatic Disease Clinics of North America, vol. 37, no. 1, pp. 33-46, 2011.

[3] L. M. Gerber, Y.-L. Chiu, M. Verjee, and H. Ghomrawi, "Healthrelated quality of life in midlife women in Qatar: relation to arthritis and symptoms of joint pain," Menopause, vol. 23, no. 3, pp. 324-329, 2016.

[4] A. C. Gelber, "Conventional medical therapy for osteoarthritis: current state of the evidence," Current Opinion in Rheumatology, vol. 27, no. 3, pp. 312-317, 2015.

[5] M. Lethbridge-Cejku, J. S. Schiller, and L. Bernadel, "Summary health statistics for U.S. adults: National Health Interview Survey, 2002," Vital and Health Statistics. Series 10, no. 222, pp. 1-151, 2004.

[6] J. Niu, Y. Q. Zhang, J. Torner et al., "Is obesity a risk factor for progressive radiographic knee osteoarthritis?" Arthritis Care and Research, vol. 61, no. 3, pp. 329-335, 2009.

[7] H. Y. Cheng, "A brief talk on contraindication of fire needle therapy," Journal of Traditional Chinese Medicine, vol. 48, pp. 303-305, 2007.

[8] V. Silverwood, M. Blagojevic-Bucknall, C. Jinks, J. L. Jordan, J. Protheroe, and K. P. Jordan, "Current evidence on risk factors for knee osteoarthritis in older adults: a systematic review and meta-analysis," Osteoarthritis and Cartilage, vol. 23, no. 4, pp. 507-515, 2015.

[9] J. W. J. Bijlsma, F. Berenbaum, and F. P. J. G. Lafeber, "Osteoarthritis: an update with relevance for clinical practice," The Lancet, vol. 377, no. 9783, pp. 2115-2126, 2011. 
[10] J. F. Innes, J. Clayton, and B. D. X. Lascelles, "Review of the safety and efficacy of long-term NSAID use in the treatment of canine osteoarthritis," Veterinary Record, vol. 166, no. 8, pp. 226-230, 2010.

[11] P. McGettigan and D. Henry, "Cardiovascular risk and inhibition of cyclooxygenase: a systematic review of the observational studies of selective and nonselective inhibitors of cyclooxygenase 2," The Journal of the American Medical Association, vol. 296, no. 13, pp. 1633-1644, 2006.

[12] E.-J. Kim, C.-Y. Lim, E.-Y. Lee, S.-D. Lee, and K.-S. Kim, "Comparing the effects of individualized, standard, sham and no acupuncture in the treatment of knee osteoarthritis: a multicenter randomized controlled trial," Trials, vol. 14, article 129, 2013.

[13] L. Qiu, J.-W. Kan, X. Zheng, M. Zhang, and J. Zhang, "Observation on the long-term efficacy of knee osteoarthritis treated with warm needling and rehabilitation training," Zhongguo Zhen Jiu, vol. 33, no. 3, pp. 199-202, 2013.

[14] J. Yu, "The rise of Chinese Acupuncture in the West: how an Ancient Eastern Tradition Became an American Medical Staple," Veterinary Heritage, vol. 38, no. 1, pp. 1-9, 2015.

[15] J.-J. Zhao, "Clinical observation of fire-needle therapy plus tuina for senile knee osteoarthritis," Journal of Acupuncture and Tuina Science, vol. 12, no. 4, pp. 260-264, 2014.

[16] T. Manyanga, M. Froese, R. Zarychanski et al., "Pain management with acupuncture in osteoarthritis: a systematic review and meta-analysis," BMC Complementary and Alternative Medicine, vol. 14, article 312, 2014.

[17] K.-D. Hong, L. Li, and X.-H. Li, "Clinical observation of warm needling therapy for treating knee osteoarthritis of different Chinese medical syndrome types," Zhongguo Zhong Xi Yi Jie He Za Zhi, vol. 32, no. 11, pp. 1466-1469, 2012.

[18] X. Li, Y. Han, J. Cui, P. Yuan, Z. Di, and L. Li, "Efficacy of warm needle moxibustion on lumbardisc herniation: a metaanalysis," Journal of Evidence-Based Complementary \& Alternative Medicine, 2015.

[19] F. Y. Fung and Y. C. Linn, "Developing traditional Chinese medicine in the era of evidence-based medicine: current evidences and challenges," Evidence-Based Complementary and Alternative Medicine, vol. 2015, Article ID 425037, 9 pages, 2015.

[20] T. Neogi, D. Aletaha, A. J. Silman et al., "The 2010 American College of Rheumatology/European League Against Rheumatism classification criteria for rheumatoid arthritis: phase 2 methodological report," Arthritis Rheum, vol. 62, no. 9, pp. 2582-2591, 2010.

[21] J. P. T. Higgins and S. Green, Corchrane Reviewers' Handbook 5.1.0, Review Manager, 2011.

[22] M. Egger, G. Davey Smith, M. Schneider, and C. Minder, "Bias in meta-analysis detected by a simple, graphical test," British Medical Journal, vol. 315, no. 7109, pp. 629-634, 1997.

[23] X. Sun, M. Briel, J. W. Busse et al., "Credibility of claims of subgroup effects in randomised controlled trials: systematic review," The British Medical Journal, vol. 344, no. 7852, Article ID e1553, 2012.

[24] M. Su, "Clinical effect of blood-letting puncture combined with red-hot needle therapy on knee osteoarthritis," Chinese Medicine Guide, vol. 34, pp. 611-612, 2012.

[25] H.-W. Zeng, B. Nie, and L.-L. Shi, "Observation on therapeutic effect of blood-letting puncture combined with red-hot needle therapy on knee osteoarthritis," Zhongguo Zhen Jiu, vol. 28, no. 7, pp. 493-495, 2008.
[26] H. Zhu, "Treatment of 56 cases of knee osteoarthritis with therapy of fire-needle and cupping," Journal of Nanjing TCM University, vol. 26, pp. 475-476, 2010.

[27] P. X. Jie, Y. Y. Peng, and H. T. Jing, "Treatment of 120 cases of knee osteoarthritis with fire needle therapy," Journal of Hebei TCM and Pharmacology, vol. 21, pp. 25-26, 2006.

[28] B. Li, X. C. Xie, and P. L. Wang, "Clinical observation of treatment on knee osteoarthritis with fire-needle moxibustion," Beijing Journal of Traditional Chinese Medicine, vol. 30, no. 12, pp. 25-26, 2011.

[29] D. J. Lu, Q. Wang, and S. W. Mei, "Analogical research on the clinical efficacy of treating knee osteoarthritis with fire needle and moxa needle," New Traditional Chinese Medicine, vol. 43, pp. 94-96, 2011.

[30] G. B. Zhang, W. L. Wang, and Q. Zhang, "Comparative study between fire needling and warm needling therapies for knee osteoarthritis," Shanghai Journal of Acupuncture and Moxibustion, vol. 32, pp. 299-300, 2013.

[31] Q. H. Kuang, "Treatment of 50 cases of knee osteoarthritis with fire needle therapy," Journal of Clinical Acupuncture and Moxibustion, vol. 22, pp. 19-20, 2006.

[32] S. P. Tao, T. F. He, and Y. B. Luo, "Observations on the efficacy of fire acupuncture plus filiform needle acupuncture in treating knee osteoarthritis," Shanghai Journal of Acupuncture and Moxibustion, vol. 32, pp. 742-746, 2013.

[33] L. Zhu, "Analogical research on the clinical efficacy of treating knee osteoarthritis with fire needle and cupping needle," Beijing Journal of Traditional Chinese Medicine, vol. 32, pp. 605-607, 2013.

[34] K. D. Huang, "Treatment of 41 cases of knee osteoarthritis with therapy of fire-needle and cupping," Chinese Journal of Geriatric Care, vol. 11, pp. 11-12, 2013.

[35] Z. Wang, "Treatment of 39 cases of knee osteoarthritis with therapy of fire-needle and Chinese medicine," TCM Research, vol. 28, pp. 45-47, 2015.

[36] J. Wu and C. Tian, "Observation on therapeutic effect of fire needle on gonarthritis of type of accumulation of pathogenic cold due to yang deficiency," Chinese Medicine Modern Distance Education of China, vol. 32, pp. 2969-2978, 2014.

[37] C. T. Chen, M. L. Chen, D. S. Tang, and S. Huang, "Clinical observation of fire needle therapy in the treatment of 50 patients of herpes zoster," Lishizhen Medicine and Materia Medica Research, vol. 18, pp. 1842-1843, 2007.

[38] F. C. Hu, B. L. Sun, C. Chen, and F. Chen, "TCM nursing of blood-letting puncture combined with red-hot needle therapy in the treatment of acute gout," Modern Journal of Integrated Traditional Chinese and Western Medicine, vol. 17, pp. 913-914, 2008.

[39] L.-Y. Yang, D.-J. Lu, and Y.-H. Li, “Observation on therapeutic effect of fire-needle therapy on lumbar intervertebral disc herniation," Zhongguo Zhen Jiu, vol. 29, no. 6, pp. 449-451, 2009.

[40] S. Lee, K. H. Kim, T.-H. Kim et al., "Moxibustion for treating knee osteoarthritis: study protocol of a multicentre randomised controlled trial," BMC Complementary and Alternative Medicine, vol. 13, article 59, 2013.

[41] H. Li, F.-H. Zhang, and Y.-C. Wang, "Observation on the efficacy of acupuncture and fire needle therapy for hand osteoarthritis," Zhongguo Zhen Jiu, vol. 33, no. 10, pp. 885-888, 2013.

[42] J. Xu, H. Deng, and X. Shen, "Safety of moxibustion: a systematic review of case reports," Evidence-Based Complementary and 
Alternative Medicine, vol. 2014, Article ID 783704, 10 pages, 2014.

[43] T.-H. Kim, K. H. Kim, J. W. Kang et al., "Moxibustion treatment for knee osteoarthritis: a multi-centre, non-blinded, randomised controlled trial on the effectiveness and safety of the moxibustion treatment versus usual care in knee osteoarthritis patients," PLoS ONE, vol. 9, no. 7, Article ID e101973, 8 pages, 2014.

[44] J. Pildal, A. Hróbjartsson, K. J. Jörgensen, J. Hilden, D. G. Altman, and P. C. Gøtzsche, "Impact of allocation concealment on conclusions drawn from meta-analyses of randomized trials," International Journal of Epidemiology, vol. 36, no. 4, pp. 847-857, 2007.

[45] D. Fanelli, "Do pressures to publish increase scientists' bias? An empirical support from US states data," PLoS ONE, vol. 5, no. 4, Article ID e10271, 2010. 


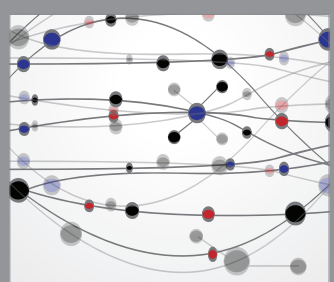

The Scientific World Journal
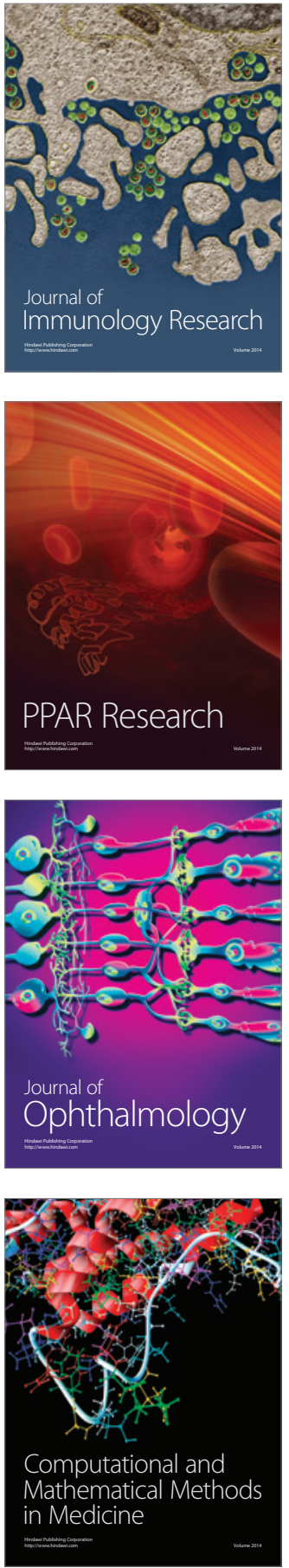

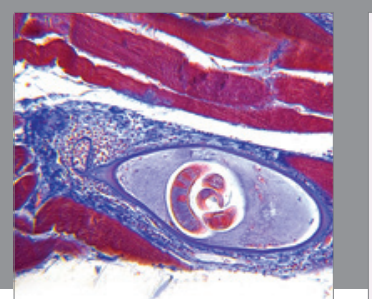

Gastroenterology Research and Practice

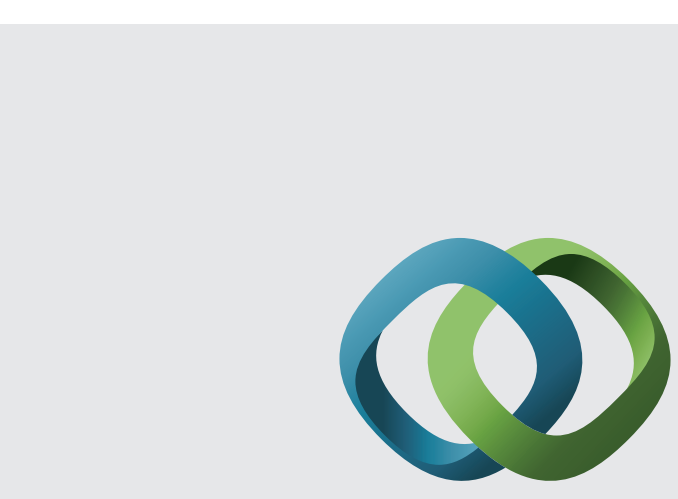

\section{Hindawi}

Submit your manuscripts at

http://www.hindawi.com
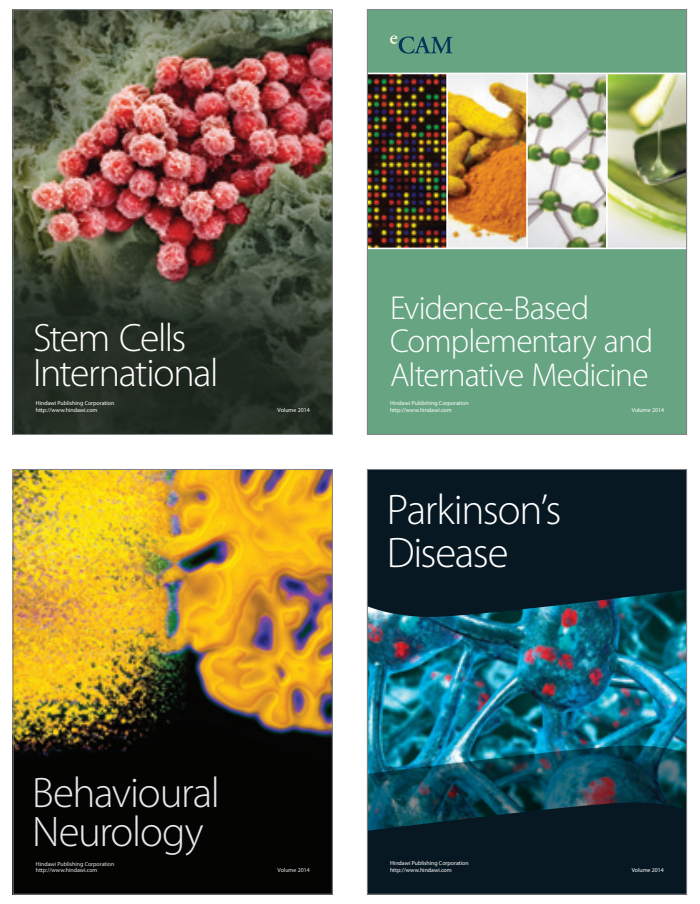
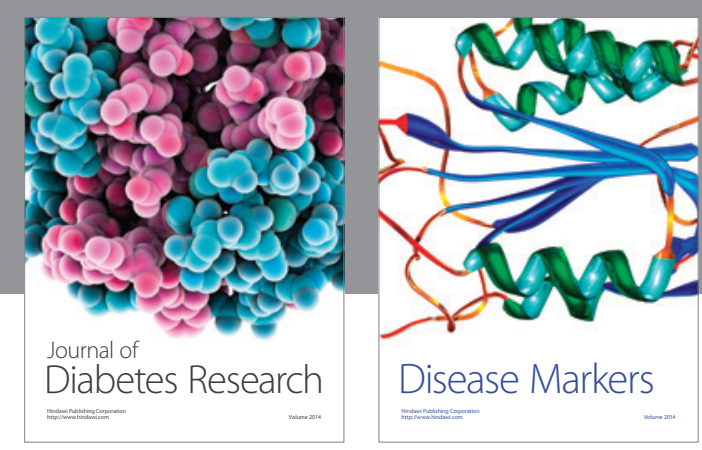

Disease Markers
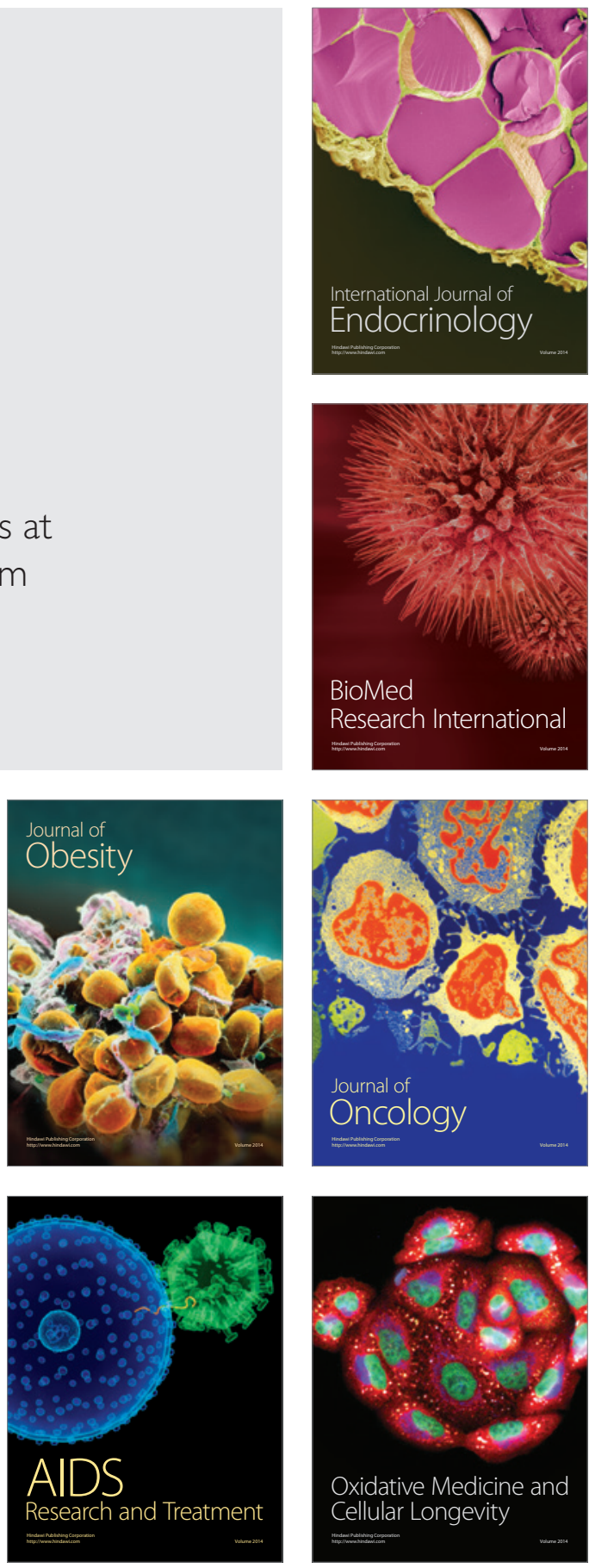